\title{
Beyond Autism Treatment: The Application of Applied Behavior Analysis in the Treatment of Emotional and Psychological Disorders
}

\author{
Robert K. Ross
}

\begin{abstract}
The field of applied behavior analysis (ABA) has increasingly come to be associated with the treatment of autism in young children. This phenomenon is largely the result of empirical research demonstrating effective treatment outcomes in this population. The same cannot be said with regard to the treatment of conditions often referred to as emotional or psychological problems. The current article describes the philosophical and descriptive differences that likely account for the lack of application of ABA in these areas and proposes potential solutions to help ABA practitioners more effectively address these issues. Specifically, the issue of how to objectively describe these "conditions" needs to be addressed so that careful study of treatment effects can occur in a manner similar to the way that brought ABA to prominence in autism treatment.
\end{abstract}

Keywords: Applied Behavior Analysis, emotional behavior, psychological disorders

\section{Introduction}

Over the course of the past decade, the field of Applied Behavior Analysis (ABA) has become synonymous in the eyes of many parents, teachers and clinicians from other disciplines with treatment of autism. Many professionals in ABA do not fully welcome a narrow view of this applied science. However, it can be argued that this is partially a very good thing for our field. The perception of ABA as the most effective treatment for children with Autism Spectrum Disorders has come about as a result of the demonstrated effectiveness of the application of the principles and procedures consistent with the science of ABA (Lovaas, 1987; NYS DPH EIP, 1999: NRC, 2001). The lack of the identification of $\mathrm{ABA}$ as the most empirically effective treatment for other areas (e.g., psychological and emotional disorders), for which is it often applied, may stem from an absence of such data and formal application of our technology. Rather than lament the over identification of ABA with autism treatment, the more adaptive response would be to conduct the kind of empirical studies in the areas of psychological and emotional disorders that has served to bring $\mathrm{ABA}$ prominence in autism treatment.

A major impediment to accomplishing this goal, in my view, is the lack of careful application of some of the tenets of applied behavior analysis to areas that are typically described as "psychological' or "emotionaP" rather than "behavioral". This needs to be addressed directly. As behavior analysts, we must be willing to use terms outside of our discipline, but insist on operational definitions for these terms when we use them. For example, a behavior analyst can treat a child who presents with a "mood disorder" by specifying the behavioralevidence of the disorder. Is it that the child is often happy, but becomes enraged when told "no" or when there is a change in their schedule? Is it that they describe high levels of variability in their mood and would like to describe more stable levels?, or it is that the child behaves in certain ways more often than we would like (hitting, yelling, inactivity,) and describe this as evidence of a mood disorder? We can, if we choose to, make specific and measureable the evidence for the disorder/diagnosis, and then apply treatment. Subsequent evaluation of levels of the symptoms can enable us to determine empirically if treatment has reduced, increased or had no effect on these symptoms. 


\section{Descriptive Differences between Behavior Analytic and Non- Behavior Analytic Approaches}

To understand why ABA based approaches to treatment of psychological and emotional issues are less well accepted, we need to describe how behavior analytic and non-behavior analytic approaches fundamentally differ in terms of how professionals talk about (describe) and treat these issues. Before doing that we must confront the elephant in the room. What is a "psychological" or "emotional' issue? This argument can be phrased as "are we treating the "emotion/psyche" or are we treating "behavior"? If we step back just a little from this question we may be able to see that the treatment goal for both perspectives is to have treatment change behavior. Unfortunately, for behavior analysts the descriptions of treatment from a non-behavior analytic framework involves the use of descriptions of hypothesized mental processes. One of the fundamental tenets of our science is that we do not embrace such hypotheticalentities such as "mind" and "will", these terms "refer to a possibly existing, but at the moment unobserved process or entity" (Moore, 1995, p.36).

While that may be how the question is correctly answered on the Behavior Analyst Certification Board exam, it is simply not what most psychologists believe and often not what some behavior analysts believe as well. Additionally the field of behavior analysis requires precise definitions and objective measurable outcomes (BACB, 2005). The treatment goal for many clinicians treating "emotional" and "psychological" issues may be that the patient self-reports to "feel better" as evidence of an improved condition. Given that the se divergent views exists, and that such terms and concepts as "mind", "mood", "anxiety", "frustration", "lack of internalization" are, and will continue to be, commonly used we must begin the process of defining what these terms and concepts mean. The gulf between behavior analytic and psychological/emotionally based treatments will likely remain large until this task is accomplished.

The requirement for precise descriptions is critical here, because it is this point that makes the distinction between behavior analytic and non-behavior analytic treatment most clear. Treatment consistent with the principles of $\mathrm{ABA}$ requires us to define in objective and reliably measurable terms, what is meant by those concepts or terms that are used to make diagnoses or describe emotional or psychological disorders. This is crucial in determining the current condition of the person whom we are treating and whether our treatment decreases symptomology and increases adaptive skills and behaviors, thereby lessening the probability of the symptoms (and thus the disorder) returning. I have purposefully assiduously avoided talking about treatment procedures thus far, because evaluating the efficacy of interventions can only happen when some agreement as to the problem being treated has been established. At this point in time, it is not clear that this condition has been met when referring to the many diagnoses of emotional and psychological disorders.

\section{Establishing Reliable Definitions for Psychological/Emotional Concepts}

Like the English and Americans, Behavior Analysts and psychologists can be said to be separated by a common language. While we both use English, we use it in remarkably divergent ways. For most psychologists the terms "mind", "mood", and "emotions" are clear and have meaning. For most behavior analysts, they represent imaginary concepts, euphemisms for observable measurable behavior, and hypotheses of causes of behavior [masquerading as a description of behavior.] This is the chasm we must overcome in order to work together effectively.

Since behavior analysts contend that these terms are faulty, we should provide some technical support to help resolve the problem. This is more productive than not bringing our technology to bear on these problems because we only want to "use our ball to play the game." I suggest several general guidelines to follow and questions to be asked that can guide our efforts to accomplish this task.

First and foremost behavior analysts need to start with a willingness to tolerate the use of common (but imprecise) language by non-behavior analysts. Behavior Analysts need to take the time to 
develop measurable definitions in conjunction with non-behavior analysts and to get agreement from them that this is what they mean by these terms. If we are going to operate in this field, we need to work effectively with those in the field. Doing so will require us to apply our principles in our interactions with other professionals. We will need to shape the behavior of others slowly and with reinforcement for any gains observed so that we can work from a common and mutually acceptable framework.

The obvious next step is to correctly describe what is being treated, and to specify the treatment goal or expected outcome. Baseline status of this condition should be specified. We need to describe what a good treatment outcome looks like in terms of adaptive functioning in all settings. For example, the child who is being seen, who takes items that do not belong to him/her (stealing), needs to be able to be alone in settings where items of interest are present and he or she does not touch or take them. For this problem, it is not enough to set a goal of having "better morals" or a "conscience". If he/she does not take items when opportunities to do so (including unobserved instances) occur repeatedly over time, we can conclude that we have taught him/her not to steal or we can infer that we have successfully supported the development of "better morals" or a "stronger conscience".

In attempting to define behavior that has historically been categorized as psychological/emotional, the symptomology that comprise the condition should always be broken into two distinct categories: Motor-physical (non-verbal) behavior and verbal behavior. If someone accomplishes the task of saying that stealing is wrong, without reductions in taking items when no-one is watching, treatment is unlikely to be described as effective. It is not enough to "know" right from wrong. One must do "right" in order for other to become convinced that the individual "knows" right from wrong. In an interesting "catch 22 " this individual is often described as lacking adequate "internalization" of the rule of not stealing, even when they can clearly state the rule. In a twist of language that Heller (1961), himself would appreciate, it turns out that evidence of "internalization" is external behavior.

Finally, we need to determine if the condition being treated occurs because the individual lacks knowledge. For example does the person "know" that most people think stealing is wrong? Or does the person "know" that people describe stealing as wrong and steal nonetheless? There is a fundamental difference between possessing information and possessing information but not engaging in the behavior consistent with this knowledge. We must accurately determine which condit ion reflects the current status of the individual before beginning to implement procedures to provide this knowledge.

Questions to be asked and answered include;

1. What does their verbal behavior indicate? (e.g., Do they report that taking other's things is not okay? If so, this evidence suggests that they understand that taking things is wrong, yet do it despite this knowledge.)

2. Will providing what they already have (knowledge of social rules and how the behavior affects others) be sufficient to diminish stealing?

3. If the answer to \# 2 is "no", then the problem (stealing) is a motor performance problem. (e.g., touching or picking up an item that does not belong to you). Simply stated, if knowledge is present and stealing persists, why do we need to continue to work on knowledge? The existence of a motor performance problem is likely to be the case in every instance of psychological/emotional conditions. It may also be true that this problem is more likely to be the failure to produce the motor skill that exists in the person's repertoire, not the lack of the motor skill. The implication of this reality is that almost all treatment for "psychological/emotional" issues must also include motor behavior change procedures, and that the use of knowledge change procedures may be superfluous. 


\section{Defining Treatment Procedures}

Precise descriptions of the desired short and long term outcomes can be a critical step in defining the treatment strategies themselves. We can define the long term goal of not stealing in the following manner: "the person will be able to be present in rooms where items that are desired are present but are not touch them". This description makes it clear that our treatment package will need to include more than discussions of why stealing is wrong and how it makes others feel. We may need to rehearse the desired (but clearly not fluent) skill of being present with items and doing something else. We may need to incorporate reinforcers to strengthen the skill/routine of not touching. We may also want to start with short durations of alone with items and systematically increase the time in the room alone based upon success in not touching items. When descriptions of what the actual physical performance looks like are used and incorporated into outcome measurement, reliance on knowledge of rules to control behavior (particularly where such reliance has proved ineffective) can be reduced. Careful review of the previous paragraph may lead the observant reader to conclude that I am suggesting an instructional program or skill practice activity rather than a traditional therapy approach to the problem of moral development or lack of internalization of rules. This reading would be correct. The response of any coach to an athlete, whose skills are not well developed, would be to suggest practicing the skill correctly so that performance of the skill under other conditions would be enhanced. Since we have identified that the problems lies more correctly in the domain of physical skill, this would seem a more reasonable response.

This is change to teaching observable measurable behavior and describing the problem in these terms rather than having a goal of "increasing the internalization of rules" is vital in that often we are treating these types of problems as if they are evidence of a disorder rather than evidence of the lack of adequate skills.

The issue of separating and working on both verbal and motor behavior is critical. When treating individuals who are verbal and possess the ability to report symptoms as well as knowledge, we must be careful to understand that verbal behavior is behavior and as a result subject to the following concerns:

1. Verbal reports of mood states, feelings, and behavior may or may not be reliable. (They may say that they "feel depressed a lot", because a recent event may result in the feeling being prevalent today but not necessarily true of how they felt the past week).

2. Reinforcement may be available for inaccurate reporting of symptomology. (They may say that they "feel depressed a lot", in order to continue to access a therapist or obtain medication)

3. Descriptions of symptomology are much more likely to be subjective unless operational definitions of these symptoms exist and are used in treatment. (They may say that they "feel depressed a lot", because they view not being "happy" all time as evidence they are depressed).

4. Adaptive verbal repertoires may need to directly teach to compete with less adaptive and well established existing repertoires. (They may say that they "feel depressed a lot", because they have not been taught other descriptors to used to describe gradations in mood).

The above is a set of guidelines that I use when asked the question "is it behavioral or psychological/psychiatric?" In fact, no question highlights the current challenge with respect to description of the problem better than this question. For many psychologists there is a clear distinction between "behavioral issues" and "psychological" or "emotional issues". While the perception may be widespread, the simple and objective reality is that almost all "psychological' issues are identified and described by evaluating behavior (including verbal behavior). We cannot know that someone is depressed unless they say and do things (complain of being depressed or stay in bed for days). If a person is active, functioning successfully every day at work and states that they are happy reliably when asked, it is unlikely that they will be considered "depressed" by most clinicians. That said, it is anathema to suggest to most psychologists that, if we increase positive statements of mood and increase activity levels and adaptive behavior at work through the use of reinforcement procedures, that the person will no longer 
be depressed. This would be dismissed as treating the symptoms rather than the "problem". The fact that the symptomology used to diagnose the condition are no longer present may be described as "not relevant" and simplistic. The larger issue of treating the "whole person" and not a collection of symptoms is raised to diminish anyone so foolish as to suggest this approach.

This mechanistic model is said to be inappropriate treatment since we are not addressing the "underlying psychological issues" that have come to cause the person to be depressed in the first place. The hypothesis that the symptomology of all psychologicalemotional issues are caused by something internal that must be worked out via specific relationship based verbal interactions is just that, a hypothesis.

An equally valid hypothesis is that the collection of observable measurable behaviors that we describe as evidence of "depression" are evidence of nothing more than that the individual engages in these behaviors. These behaviors can be decreased by providing identified reinforcers to competing adaptive skills and limiting reinforcement when the behaviors that comprise the symptoms of depression occur.

The problems of the traditional psychological approach here for behavior analysts are many.

The paradigm of emotion and past trauma causing psychological disorders is a circular one. The circle goes like this, past events make you "upset", and this causes you to experience "distress" that "makes" you have "difficulty" in the same or similar situations in the future. This account makes you a victim of past events and "explains" why you are sad, angry, or mad. It is an account that diminishes or eliminates the role of learning in the account of why new skills, repertoires of behavior and language are acquired and maintained.

Behavior is viewed by traditional psychologists as a symptom of a disorder or condition not a functional response that enables you to escape, avoid or mitigate exposure to this unpleasant condition. A corollary of this hypothesis is that maladaptive behavior is not functional Another version of how this mechanism is used is the explanation for the condition of Reactive Attachment Disorder (RAD): The failure to have "bonded" or "attached" to a parent/primary caregiver is the "reason" why a child treats adults poorly, steals or seeks attention from current caregivers. The idea that these behaviors could produce responses from current caregivers that are desired by the child (and thus these behaviors are being reinforced by those caregivers) is not taken into account, only that past failure to appropriately "attach" explains these behaviors. If instead we describe what the child does and does not do, and what specific skills need to be developed, we can begin the process of effective treatment.

It may be that a desired skill is to demonstrate emotion consistent with events (cry when talking about past events of a sad nature rather than laugh). This is a motor task, not a verbal performance, and therefore can be practiced. The absence of this performance can be viewed as a psychological deficit and/or evidence of a disorder or it can be seen as a skill that is not currently demonstrated.

It is in this view that ABA may have much more to offer treatment for areas historically viewed as out of the province of ABA. The absence of effective instruction to reinforce adaptive social behavior and the presence of reinforcement for maladaptive (but functional) behavior accounts for the presence of those specific topographies of behavior in young children. As a behavior analyst you can recognize that past trauma may evoke behavior, but you know that whether those specific topographies of behavior continue or diminish is the result of consequences in the current environment.

However, this view is not the dominant one in our culture. Practitioners in the fields of psychology and psychiatry are still primarily attempting to treat behavior by treating the "mind". These 
attempts are firmly rooted in a belief that changing "thought" is necessary and at times sufficient to change behavior. The evidence of this change is often a change in verbal behavior.

\section{A Focus on Language:}

As I have previously stated, language is often at the heart of our differences in approach to treatment. As behavior analysts, we look for precision in descriptions of human behavior and instead we find euphemisms replete in psychological literature. Terms that have no single agreed-upon meaning are rampant in the literature on the treatment of psychological and emotional issues. These devices we create often "mis-describe" what is actually happening and unfortunately this practice often interferes with, if not prevents, effective treatment.

Examples of problematic descriptions include such terms as: emotional states (i.e. "angry", "frustrated", "insecure", "anxious"); issues (i.e. "self-esteem issues", "transition issues", "sensory issues", "executive functioning issues"); diagnoses (i.e. RAD, ADHD, Bi-Polar Disorder); and conditions (i.e. "lacks a conscience", "shows no remorse", "does not accept responsibility for his/her actions").

All of these reflect our interpretations of what they do, without accurately describing behaviors. The terms often represent a hypothesized "why" or cause of the behavior that they purport to describe. They are interesting yet unhelpful and may be highly misleading. It is often the case that a child who is seeking parental attention may engage in aggression. They do so when attention is less available or because doing so under similar stimulus conditions has been reinforced in the past. Was the child who was aggressive angry? Was he/she frustrated? Or perhaps he/she were internally experiencing some past trauma that was triggered by a sound in their environment and this caused the child to lash out protectively?

We can apply our clinical judgment and decide that since they hit the parent, they were angry. But can we truly know that this is true? Since our interpretation is just that, an interpretation, others may interpret the situation from an entirely different point of view. Can accurate treatment flow from a model that is completely subjective and based upon the point of view of the person doing the interpreting? This model makes the diagnostic focus the clinician, rather than the person who is being treated. Simply by shifting from an objective description of the behavior occurring to a subjective categorization of the behavior (complete with hypothesized cause), we create a pathology and a rationalization that flows directly from our subjective interpretations. While objective descriptions of behavior may be far less interesting and explanatory, they do have the benefit of being accurate regardless of the observer. This should be a minimum criterion prior to beginning treatment.

Perhaps the child in the above scenario can provide insight into his or her behavior. This might be of significant help provided the child is an accurate reporter and is capable of such insight. However without such assistance, it is still possible to determine if the child is or is not provided attention from parents subsequent to aggression and if this functions to maintain the behavior. It is also possible to provide attention for more adaptive interactions and reduce levels of attention subsequent to aggression. If attention truly was the reinforcer maintaining aggressive behavior, effective treatment can occur without resorting to the use of euphemisms.

Similar circular arguments have also been used with a wide range of "emotional behaviors". If someone is "yelling" and we decide that this means that they are frustrated, we must understand why they are frustrated and "teach" then to tell us they are frustrated with words we like better. However, this may not address the problem, if they are yelling to get a task removed and it works, then telling us that they are frustrated with words, (without the task being removed) does not help them. It makes them likely to continue to yell to make the task go away. This interestingly presents another "catch 22", since the previous treatment did not successfully resolve their "frustration issues", they still present as "frustrated" 
and it is now inferred as meaning that these frustration issues are "deep seated" thus requiring more "therapy" to get to the "real root" of the problem.

The good news is however that it is not too difficult to parse out the behaviors from which these euphemisms were comprised and to obtain reliable descriptions for use in treatment. It does not necessarily mean abandoning these euphemisms, but simply defining what they mean for a particular person by describing the behaviors that lead to that label for that particular person. For example, before we can say a child has "sensory issues" we will have to describe the fact that when certain noises (e.g. loud sounds and the telephone ringing) occur in the environment, the child puts his/her hands to his/her ears. If this is what we mean by sensory issues, then why don't we just say so? The term is so broad that it may be assumed that the child has a wide range of problems not in evidence. It also implies that the cause has been investigated and proven to be "sensory". "Sensory" is not a medical cause, it is a euphemism to describe a broad range of possible symptoms but nothing specific.

Other examples of the different perspective of two common descriptions include: "lack of conscience" and "poor self-esteem". The psychological perspective of "lack of conscience" is that the child fails to feel guilty when he/she has done something "immoral". Something is missing, likely due to some early deficit in the child rearing environment, or trauma occurring at that time. The behavioralperspective involves a description which defines that the child does things that we do not like and we describe as inappropriate; for example, laughing when another child is injured. The psychological perspective of "poor self-esteem" is that the child views him/her self negatively or does not "value" him/her self as a person .The behavioral definition is that the child makes self-deprecating statements and/or engages in behavior that we do not like (because it is unsafe or socially not valued); for example, saying "I'm stupid" or "I can't do this".

The traditional approach is that the solution to the above problems or condition lies in getting the child to have a better understanding of the past. This way the child can know why he/she makes bad decisions or why doing so is not under his/her control, and what he/she can do differently in the future (this approach presumes the child does not have this knowledge currently). If this does not help or while this help is ongoing proponents of this approach may suggest prescription medications for this condition. A tenet of this approach is that the problem and its causes are very complex and thus treatment is often not readily accessible and effective in ameliorating the problem.

As a behavior analyst, I fundamentally reject this premise and offer an alternative. First, reduce complexity by providing objective descriptions of specific behaviors, then build ing more complex behaviors by establishing simple skills and expanding on those basic skills.

The best analogy I have to offer is how we teach math and money skills. We do not begin by working on concepts. We begin by teaching counting and 1:1 correspondence. On these simple skills we build addition and subtraction. The process of increasing complexity expands to algebra and trigonometry. A person who cannot identify nickels from dimes is unlikely to understand Keynesian economic theory. However does someone who can use money functionally, make purchases, count change, and keep a checking account, truly understand money?

A person who laughs when another person is injured may be described as lacking empathy and considered to have problems "connecting with others" and thus may be labeled as having RAD, a "complex condition". However is this the problem or is it that they have not learned to respond to injuries by asking if the person needs help or by saying "Are you OK?"

The point here is that the problem is one of skill deficits and the solution should be one of skill development. In the previous scenario, we can development the following skill development plan: 
1. Teach the child to visually discriminate "sad" from "happy" and to say "What's' wrong?" to the sad model. If the child does not say such things or visually present as concerned then he/she will not be considered by objective observers to demonstrate "empathy". If the child can "empathize" but does not demonstrate the above performances (in the correct contexts) then no one will know he/she has empathy.

2. Systematically generalize the demonstration of this skill in progressively more naturalistic conditions (ensuring that correct demonstrations are reinforced). This is critical because if the child can demonstrate all of the correct performances but does not do so under appropriate contextual control he/she is likely to be viewed with some such label as "mean spirited" or "cruel".

I believe that it is imperative that behavior analysts move beyond autism and apply our technology to a broader range of conditions. In order for this to occur, we will need to work diligently and collaboratively with other professionals to remove the artificial barriers between what are considered to be "psycholo gical conditions" and what are considered to be "behavioral issues". We must all understand that there are no emotional disorders that do not involve behavior. If behavior analysts are to work with psychologists to address emotional behavior they must do so by establishing precise criteria for the use of terms to describe emotional behavior. These criteria would need to be observable and measurable and have acceptable inter-observer reliability. Skill building procedures can then be developed from these descriptions. This process is a necessary first step that will enable more objective research of behavioral analytic treatments for these conditions. The widespread application of behavior analysis to the treatment of emotional or psychological conditions is unlikely to occur unless and until these critical initial actions occur. However, once this has occurred, research similar to the type that Lovaas and Smith (1987) conducted with children with autism can be done in order to have a similar impact on the treatment of children with emotional or psychological problems.

\section{References}

Behavior Analyst Certification Board. (2005). Behavior analyst task list, third edition.

Tallahassee, FL: author. Retrieved October 10, 2007, from

http://www.bacb.com/consum_frame.html

Butter, E. M., Wynn, J., Mulick, J.A. (2003). Early Intervention critical to autism treatment. Pediatric Annals. Thorofare:. Vol. 32, Iss. 10; p. 677

Heller, J. (1961). Catch-22. New York: Simon

Lovaas, O.I. \& Smith,T. (1987). Intensive behavioral treatment for young autistic children. In

B.B. Lahey \& A.E. Kazdin (Eds.), Advances in clinical child psychology (Vol.11,pp.285-

324). New York: Plenum

Moore, J. (1995). Radical Behaviorism and the subjective-objective distinction. The Behavior Analyst, $18,33-49$

National Research Council (2001). Educating Children with Autism, Committee on Educational Interventions for Children with Autism, Division of Behavioral and Social Sciences and Education, Washington, D.C.: National Academy Press.

http://books.nap.edu/books/0309072697/html/index.html 
New York State Department of Health Early Intervention Program (1999). Clinical Practice Guideline: The Guideline Technical Report, Autism/Pervasive Developmental Disorders, Assessment and Intervention for Young Children. Publication \#4217. Health Education Services, P.O. Box 7126, Albany, NY 12224.

Author Contact Information:

Robert Ross

BEACON Services

321 Fortune Blvd. Milford MA 01757

508-478-0207 Ext 240

Cell: 508-265-3821

\title{
Behavior Analyst Online
}

\author{
www.Behavior -Analyst-Online.org
}

The Behavior Analyst Online organization (BAO) develops and deploys new resources for behavior analysts and makes them available on the Internet free of charge to the public. These resources are dedicated to educating the public about behavior analysis as well as serving as a resource for professionals involved in the field of behavior analysis.

The BAO organization is responsible to its membership to develop resources that the membership will find useful in everyday research, education, and application of the science of behavior analysis.

The BAO organization offers may perks to its members, including a Web Forum and the ABA-PRO Mailing List. In addition, the organization publishes several major free e-journals of interest to the behavior analysis community:

The Behavior Analyst Today

The Journal of Early and Intensive Behavior Intervention

The International Journal of Behavioral Consultation and Therapy

The Journal of Speech and Language Pathology - Applied Behavior Analysis

The Behavioral Development Bulletin

The Journal of Analysis of Gambling Behavior

Membership in the BAO organization is free. For details, visit our website at www.behavior-analyst-today.org 